\title{
SIMULTANEOUS OBSTACLE DETECTION FOR MOBILE ROBOTS AND \\ ITS LOCALIZATION FOR AUTOMATIC BATTERY RECHARGING
}

\author{
*Sang-Il Gho*, Jong-Suk Choi*, *Ji-Yoon Yoo**, Mun-Sang Kim* \\ * Department of Electrical Engineering Graduate School, Korea Univ. \\ Intelligent Robotics Research Center Korea Institute of Science and Technology \\ E-mail : *kosi,\{cjs,munsang\}@kist.re.kr \\ **jyyoo@elec.korea.ac.kr
}

\begin{abstract}
In this paper, we will research the simultaneous performing of both the obstacle detection for mobile robots and the localization for automatic battery recharging. And also, this research suggests the new-concept of sensor system using the ultrasonic devices for performing the above mentioned tasks. Robots with this new sensor system can not only easily detect the obstacles when moving, but also know their own relative position to the recharging station. For the performance verification, the proposed system will be applied to the home service robot, called 'HOMBOT II' which was developed by Intelligence Robotics Research Center at KIST(Korea Institute of Science and Technology).
\end{abstract}

Keywords : Obstacle detection, Localization, Mobile robot

\section{INTRODUCTION}

The powerful and long-last power system for mobile robots is needed to perform the given tasks smoothly and perfectly. Unfortunately, there are the limitations of battery technology. And also, when we use the recharging system, the recharging battery should be replaced and recharged manually by human being. So, many autonomous recharging systems of mobile robots have been developed to solve these problems. In this paper, the localization system using the existent ultrasonic system implemented in 'Hombot II' for the obstacle detection is suggested to perform the docking task to the station for the automatic recharging. Robot with the suggested new sensing system can have not only the ability to detect the obstacle but also to estimate its relative position to the station. The suggested sensing system has a merit in the aspect of the cost comparison with other sensing systems for the localization because it uses just the ultrasonic sensor.

\section{THE OBSTACLE DETECTION}

\subsection{The basic concept and structure of the obstacle detection system}

Hombot has already been equipped with the 12 us(ultrasonic) modules in the front, the back side, the right side and the left side for the obstacle detection (Figure 1). While moving, Hombot can detect the obstacles using the 12 us modules. To avoid the cross-talks between modules, the 12 us modules are divided into the 4 groups, and then the 4 groups consecutively carry out their ultrasonic emitting and receiving task.
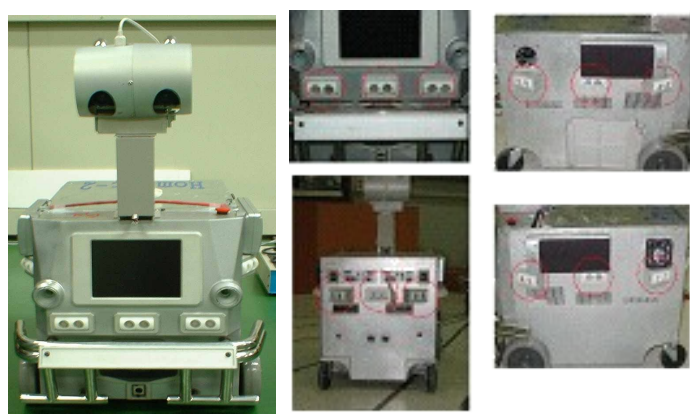

Figure 1. Hombot and the us modules' configuration

The obstacle detection system is composed of the main control board and 12 us modules (Figure 2). The main control board caculates distances between us modules and obstacles through the ultrasonic transimitting and receiving operation, and then transmits the distance data to the main computer of hombot using the CAN-based communication.

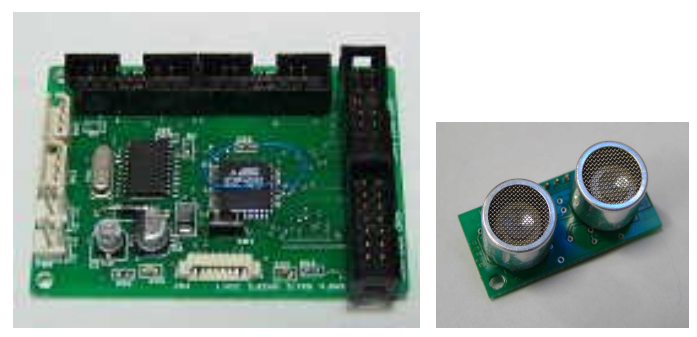


Figure 2. the main control board and the us module

\subsection{The distance measurement using us modules}

The main control board of Hombot sends the trigger pulse into the us module, and then the us module emits the ultrasonic bursts and waits for the reflected ultrasonic. If us module receives the reflected signal, us module sends the echo pulse signal for the us reception into the main control board. That is, if the us modules gets the trigger pulse from the main control board, it pulls up the level of the echo pulse output to be ready to receive the reflected ultrasonic. Then, when the reflected ultrasonic signal is received, the level of the echo pulse output is pulled down. The distance could be measured by using two time points at which the signal level of the echo pulse output is pulled up and pulled down with considering the speed of sound in air. Figure 3 illustrates the operating principle of the us module.

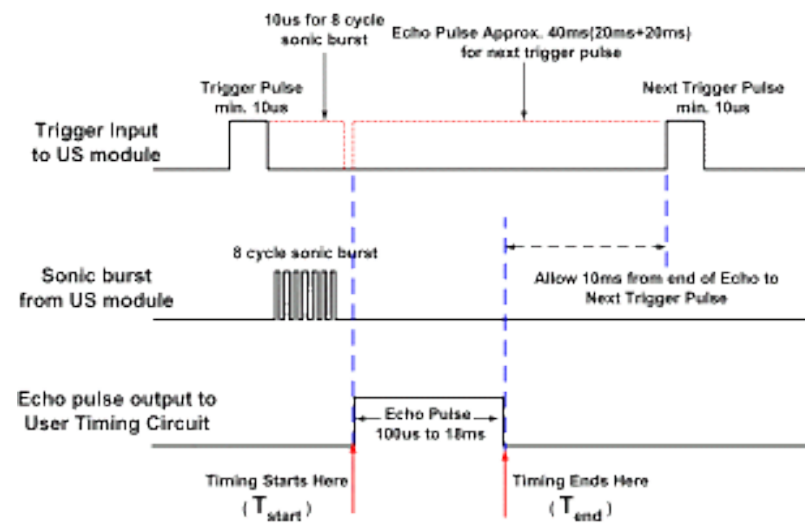

Figure 3. the operating principle of the us module

\section{THE LOCALIZATION SYSTEM}

\subsection{The basic concept of the localization system}

The localization system is developed by modifying the existing ultrasonic system for the obstacle detection. The main purpose of this research is to develop the integrated system which is capable of the obstacle detection and the localization of robot just using the ultrasonic sensor without any other additional sensors. Figure 4 shows the localization of robot. Both receivers of us module $\# 0$ and $\# 2$ of Hombot receive the ultrasonic signals emitted sequentially from two transmitters of the external station. After that, the localization system gets the four distance data. Using the four distance data - Dist at \#0 from Tx1, Dist at \#1 form Tx1, Dist at \#0 from Tx2 and Dist at \#1 from Tx2, the relative coordinates of two receivers of us module \#0 and \#2 of Hombot can be calculated.

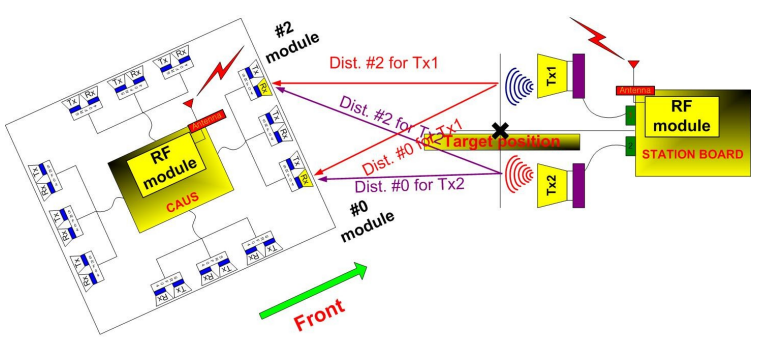

Only two modules(\#0 and \#2) receive Ultrasound transmitted from $\mathrm{Tx} 1$ and $\mathrm{Tx} 2$

Figure 4. the localization system

\subsection{The composition of the localization system and the calculation of the position}

(1) The main control board for Hombot

The main control borrad (Figure 5) enables Hombot both basically to detect the obstacles while moving and to estimate the relative position of Hombot to the station. And it transfers the modes of us modules according to the kind of operation which is the obstacle detection or the localization. When Hombot operates the obstacle detection, the main control board uses the us modules as the mode of us transmission-recption. On the other hand, when Hombot operates the localization, the us module is changed into the mode of us reception only.

The main control board has the RF module to communicate with the station, and it uses the CAN based communication with the main computer of Hombot.
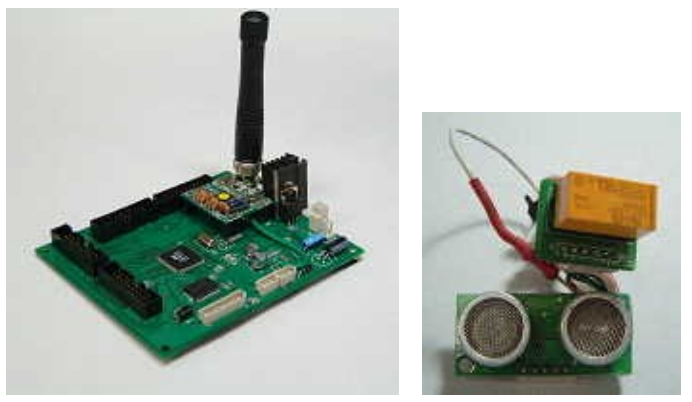

Figure 5. the main control board for Hombot and the modified us module

(2) The modified us module

The us modules is modified to be suitable for the mode change. Figure 6 is the schematic diagram of the revised transmission part of us module. In the modified system, handling the relay makes it posible to control for the transmission part of us module to be turned on or off. If the transmitter of us module is off for the mode of localization, the us module just 
can be ready to receive the externally emitted ultrasonic. On the other hand, if the transmitter of the us module is on for the mode of obstacle detection, the us module can do not only transmitting us but also receiving us which is emitted by its own transmitter.

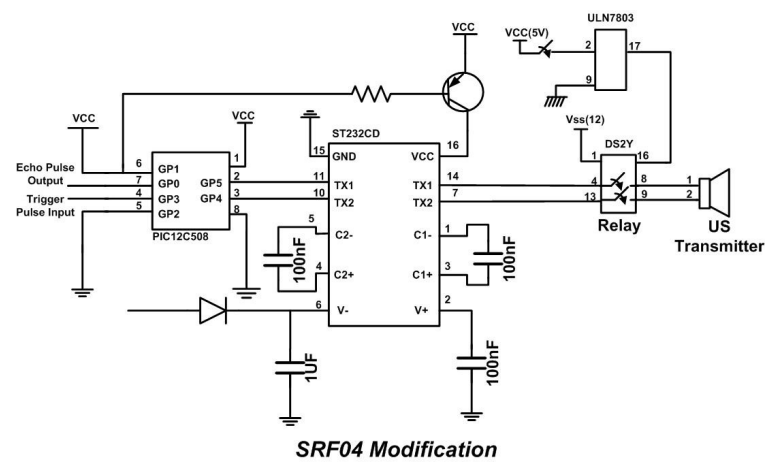

Figure 6. the schematic diagram of the modified us module

(3) The external us transmission system of the station

The external us transmisstion system for the station is composed of the us transmission control board and two us transmitters (Figure 7).
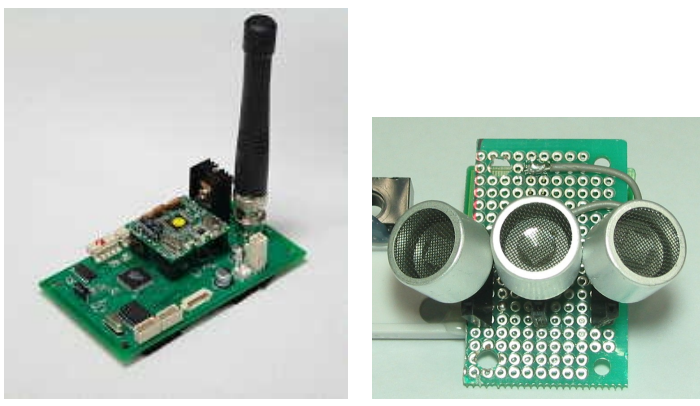

Figure 7. the external us transmission system

The us transmission control board chooses the method of us emission according to the kind of command transmitted from the main control board of Hombot through the RF communication.

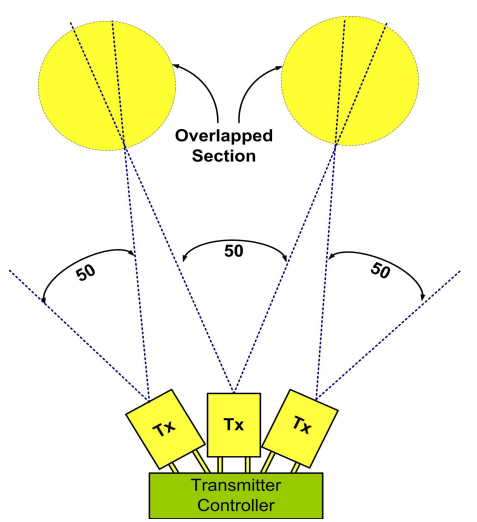

Figure 8. the extension of directivity

The us transmitter of the station emitts the $40 \mathrm{kHz}$ us and has the directivity of 50[deg.]. The us transmitter has to be modified like the right picture of Figure 7 to expand the directivity in order to overcome the limitation of the angle of us transmission-reception owing to the small directivity of 50[deg.] (Figure 8).

(4) How to calculate the position of Hombot's receivers, $\mathrm{Rx} 0$ and $\mathrm{Rx} 2$.

The positions of two receivers, $\mathrm{Rx} 0$ and $\mathrm{Rx} 2$ of Hombot, can be calculated by using the equation of circle with the four distance data (Figure 9).

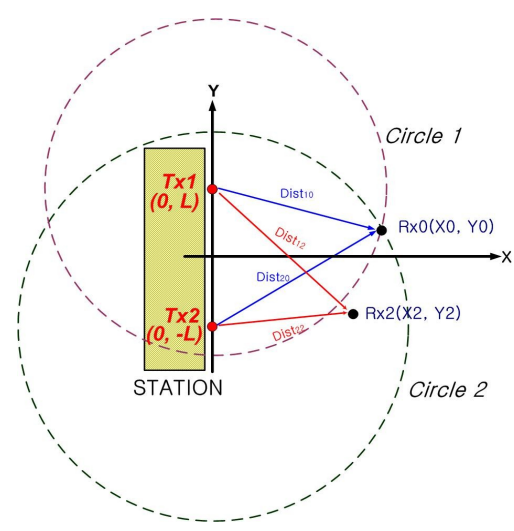

Figure 9. Getting positions using the equation of circle.

$$
\begin{aligned}
& X 0=\sqrt{\text { Dist }_{10}{ }^{2} \quad(y \quad L-)^{2}} \quad \sqrt{\text { Bist }_{20}{ }^{2} \quad(y \neq L)^{2}} \\
& Y 0=\frac{\text { Dist }_{10}{ }^{2}-\text { Dist }_{20}{ }^{2}}{-4 L} \\
& \left.X 2=\sqrt{\text { Dist }_{12}^{2} \quad\left(\begin{array}{ll}
y & L
\end{array}\right)^{2}} \quad \sqrt{\text { Aist }_{22}{ }^{2} \quad(y=L}\right)^{2} \\
& Y 2=\frac{\text { Dist }_{12}{ }^{2}-\text { Dist }_{22}{ }^{2}}{-4 L}
\end{aligned}
$$


The coordinates of two receivers are the above formulas (1), (2), (3) and (4).

\section{TIME SYNCHRONIZATION}

The start time of us reception of Hombot $(=t 1)$ has to coincide with the us transmission time of the station $(=\mathrm{t} 2)$ in order to get the exact distance data between Hombot and the station. The method to coincide $\mathrm{t} 1$ with $\mathrm{t} 2$ is named 'time synchronization'. There are no ways for Hombot to communicate with station except for RF communication. And there is the fixed delay time between Hombot's RF module and station's RF module when the transmited RF packet from Hombot is received to the RF module of the station. However the process time of RF packet computed by the micro-processor of station's control board is not fixed but always varied. Thus, Hombot successively transmits two RF packets, 'the command packet' and 'the sync Packet'. The command packet means the us transmission method of the station. The sync packet is actually used for time synchronization. The station understands the meaning of the first RF packet (the command packet) through processing the command packet using the micro-processor. After the enough processing time of the command packet, which is experimentally selected and fixed, the station is ready to receive the second RF packet (the sync packet). The sync packet through the RF module of the station is transmitted not to the micro-processor, but to the simple circuit of the control board of the station (Figure 10).

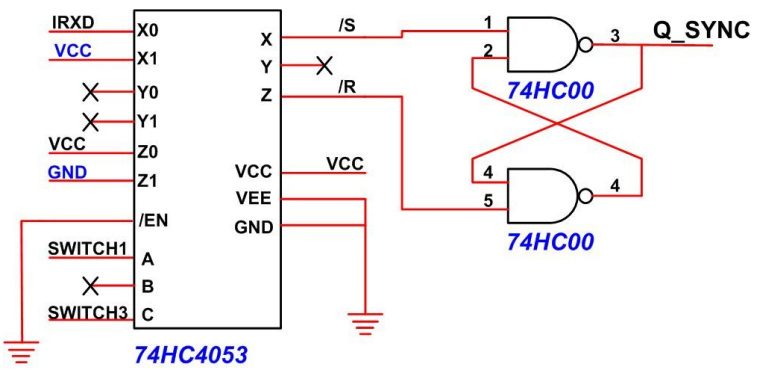

Figure 10. the circuit for capturing the sync packet

After the enough processing time for the command packet, the signal line of RF packet is connected to the input pin (IRXD) of the multiplexer 74HC4053 instead of the micro-processor of the station.

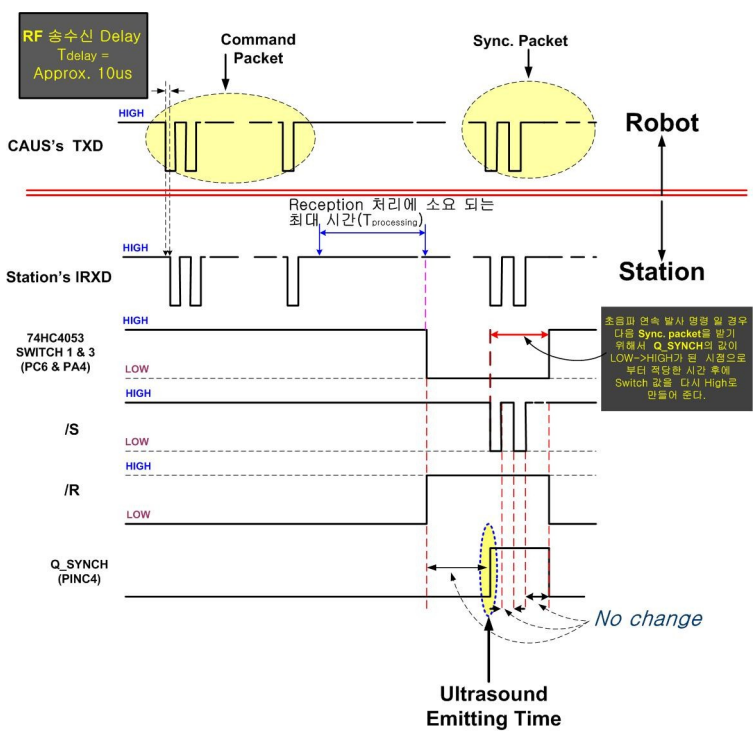

Figure 11. the timing diagram for the time sync.

Thus, if the station's control borrad receives the sync packet through the RF module, it can capture the exact time at which the sync packet is transmitted to the input pin(IRXD) of the above circuit. When the sync packet enters the input pin(IRXD), the level of 'Q_SYNC' changes 'Low' into 'High'. The fixed delay time for the RF communication can be compensted because the communication time between two RF modules is fixed. If the fixed delay time is compensated, the us transmission start time can be synchronized with the us reception start time of Hombot by capturing the time point at which the level of 'Q_SYNC' changes 'Low' into 'High'. Figure $\mathbf{1 1}$ is a timing diagram for the time synchronization.

\section{THE EXPERIMENT AND RESULT}

The localization system is taken into Hombot, and then the performance of the localization system has been tested using a GUI developed for various experiments (Figure 12).

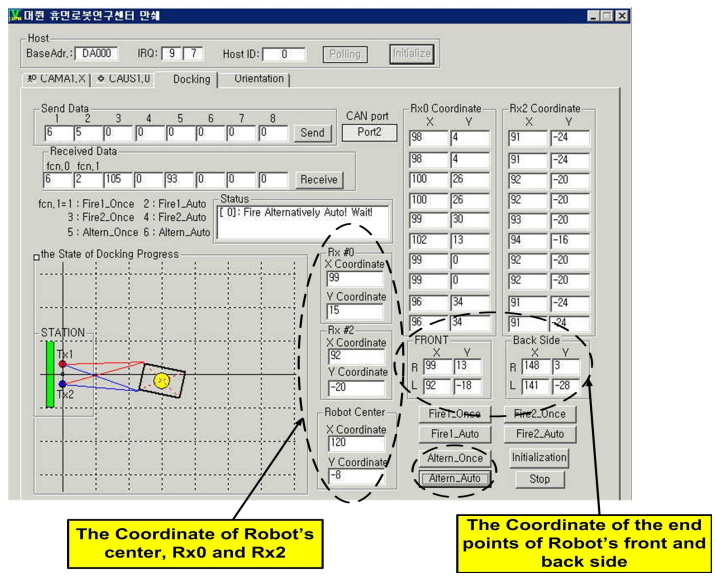


Figure 12. the GUI for the localization

The relative position of Hombot to the station has been estimated through the GUI after positioning Hombot like Figure 13.

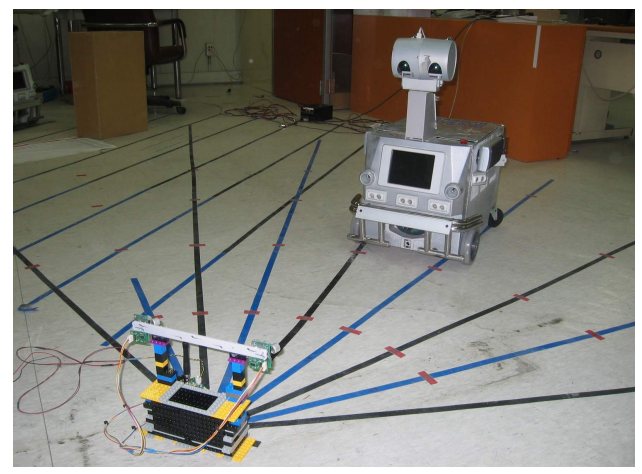

Figure 13. the experimental circumstance

The measured positon by the localization system has been compared with the real position acquired by the 3 -D coordinate mearsuing equipment named 'Flash point' (Figure 14).

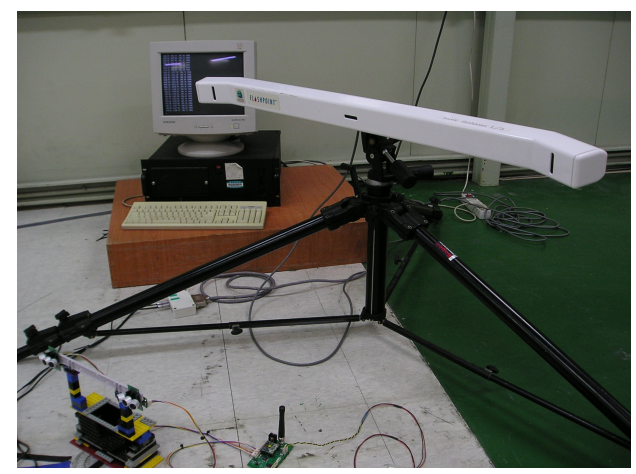

Figure 14. Flash point

In our experimental results, the average of error was incresed as much as Hombot becomes more distant from the station (Figure 15). The reason may arise from the fact that we have used the fixed value of velocity about the ultrasonic signal $(340 \mathrm{~m} / \mathrm{s})$ while it could be affected by the external conditions of environment such as air pressure, humidity, temperature and so on.

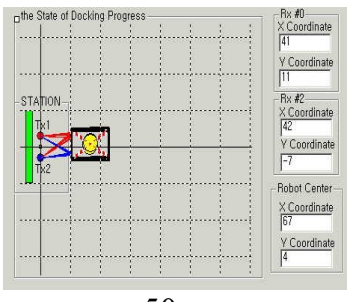

$50 \mathrm{~cm}$

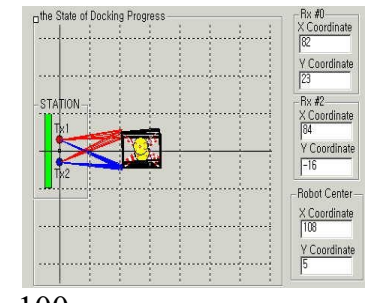

$100 \mathrm{~cm}$

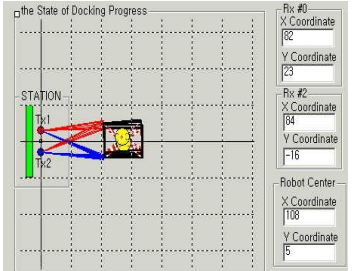

$150 \mathrm{~cm}$

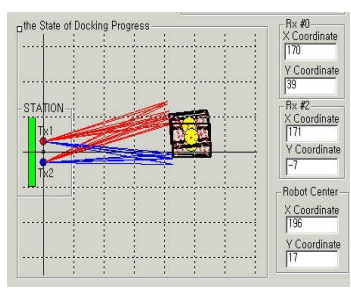

$200 \mathrm{~cm}$

\begin{tabular}{|c|c|c|c|c|}
\hline Distance & $50 \mathrm{~cm}$ & $100 \mathrm{~cm}$ & $150 \mathrm{~cm}$ & $200 \mathrm{~cm}$ \\
\hline The aver. error & $4.5 \mathrm{~cm}$ & $8.6 \mathrm{~cm}$ & $19.4 \mathrm{~cm}$ & $27.2 \mathrm{~cm}$ \\
\hline
\end{tabular}

Figure 15. the result of localization for the distance

And also, though the us transmitter is modified to overcome the limitation of us directivity, there still exists the range of angle at which the localization can not be achieved, the angles over $45^{\circ}$ on the basis of the centered line with the distance of $50 \mathrm{~cm}$ from the station. And the intensity of us emission has the difference according to the angle (Figure 16). The difference of intensity can also affect the precision of localization.

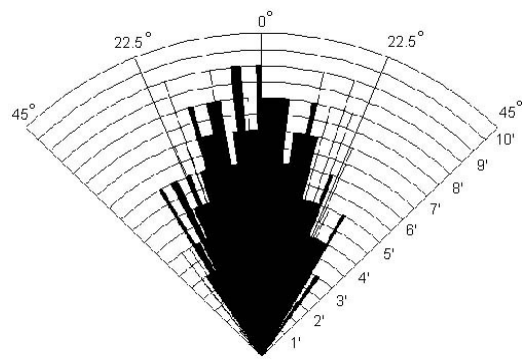

Figure 16. the intensity pattern of the us transmitter

\section{THE CONCLUSION}

Hombot already has the ultrasonic system for the obstacle detection. The existing ultrasonic system is modified to develop the localization system with the obstacle detection for practical use. The new ultrasonic system simultaneously have two functions of the obstacle detection and the localization. That is, the mode of the ultrasonic system can be changed according to the main purpose which is to detect the obstacle or to estimate the relative position of robot. When localizing robot, the average of error between the real position and the measured position increases as much as the robot is put more and more distance from the station. And there still exists the range of angle at which robot can not estimate its own position despite of modifying the us transmitter for overcoming the limitation of us directivity. In advance, the further study to overcome the weak points of ultrasonic system such as the directivity, the difference of intensity and so on is needed to enhance 
the performance of the suggested system. Moreover, the real docking needs to be experimented using the localization system.

\section{REFERENCES}

[1] C. Wattanasin., Y. Aiyama., D. Kurabayashi. "A Hybrid Technique to Supply Indoor Service Robots," IEEE International Conference on Robotics and Automation, pp. 89-94, May 1998.

[2] Zhao Feng-ji, Guo Hai-jiao and Kenichi Abe, “A Mobile Robot Localization Using Ultrasonic Sensors in Indoor Environment", IEEE International Workshop on Robot and Human Communication, pp.52-57, 1997.

[3] Milo C. Silverman, Dan Nies, Boyoon Jung and Gaurav S. Sukhatme "Staying Alive : A Docking Station for Autonomous Robot Recharging," IEEE International Conference on Robotics and Automation, pp. 1050-1055, May 2002.

[4] P. Mira Vaz, R. Ferreira, V. Grossmann, M. I. Ribeiro, "Docking of a mobile platform based on infrared sensors," IEEE Catalog Number : 97TH8280, pp. 735-740, ISIE 1997.

[5] Tong feng, Xu tian zeng, "An Ultrasonic Navigation Automatic Guided Vehicle System used in CIMS," Proceedings of the 4th World Congress on Intelligent Control and Automation, pp. 2645-2649, June 2002.

[6] Li Zhang and Bijoy K. Ghosh, "Line Segment Based Map Building and Localization Using 2D Laser Rangefinder", Proceedings of the 2000 IEEE International Conference on Robotics \& Automation San Francisco, pp. 2538-2543, April 2000.

[7] Zhao Feng-ji, Guo Hai-jiao and Kenichi Abe, "A Mobile Robot Localization Using Ultrasonic Sensors in Indoor Environment", IEEE International Workshop on Robot and Human Communication, pp.52-57, 1997.

[8] Youngjoon Han, Hernsoo Hahn, "Loclization and Classification of Target Surfaces Using Two Pairs of Ultrasonic Sensors", Proceedings of the 1999 IEEE International Conference on Robotics \& Automation Detroit, Michigan, pp. 637-643, May 1999.

[9] Dirk Bank, "A Novel Ultrasonic Sensing System for Autonomous Mobile Systems", IEEE Sensors Journal, Vol. 2, No. 6, December 2002.

[10] J. Neira et al.,"Multisensor Mobile Robot Localization", IEEE Int. Conf. on Robotics and Automation, vol. 1, pp. 673-679, April 1996.
[11] V. Gabbani et al.,"Multielement Ultrasonic System for Robotics Navigation", IEEE Int. Conf. on Robotics and Automation, vol. 3, pp. 3609-3614, April 1995. 